\title{
Le Commerce de L'Edition Musicale Française au XIXème Siecle Les chiffres du déclin
}

\author{
Anik Devriès-Lesure
}

L'aspect commercial de l'édition musicale n'a pas, jusqu'à présent, beaucoup retenu l'attention des chercheurs. Si l'on dispose maintenant de bonnes monographies concernant quelques grandes maisons européennes, on n'a qu'une idée assez vague de la manière dont elles se partageaient le marché mondial et de l'évolution de l'offre et de la demande. L'une des raisons de cette lacune est la difficulté de trouver des sources fournissant quantitativement les mouvements de partitions d'un pays à l'autre.

Pour la France, les registres tenus à partir de 1827, par l'administration des douanes, dans lesquels sont consignées, année par année, toutes les entrées ou sorties de marchandises sur notre sol, livrent des renseignements précieux sur ces échanges. Ces consignations ont été publiées dès le XIXème siècle, selon un rythme décennal, șous le titre de : Tableau décennal du commerce de la France avec ses colonies et les puissances étrangères (périodes 1827-1836, 1837-1846, 1847-1856, 1857-1866, 1867-1876, 1877$1886,1887-1896)$. Les éditions musicales, au même titre que d'autres denrées (chapeaux de paille, peaux et fourrures, lithographies, cartes à jouer, livres, cartes de géographie, ou instruments de musique ${ }^{1}$, ont fait l'objet de tels enregistrements, sous une rubrique intitulée indifféremment soit "musique gravée", ou "musique gravée ou imprimée"2. L'unité de mesure choisie par 
l'administration des douanes pour quantifier les transactions propres à la musique était le poids en kilogrammes, avec une mention, en complément, de la valeur monétaire correspondante. Aucun signalement du type des oeuvres ayant fait l'objet des échanges n'est donné. Si l'étalon poids s'applique mal au commerce de la musique, il offre au moins un avantage, celui d'être une valeur constante, qui permet de suivre, au fil des ans, l'évolution quantitative des échanges commerciaux. La valeur monétaire étant, quant à elle, beaucoup moins fiable, puisque soumise aux fluctuations économiques et au phénomène d'inflation ${ }^{3}$.

Le contenu de ces registres, bien que partiel, puisqu'aucune information n'est livrée sur les catégories musicales ayant fait l'objet des échanges et que le suivi des transactions est parfois fragmenté dans le temps, livre néanmoins une image globale du développement du marché de la musique entre 1827 et 1896. Il révèle aussi l'amplitude de certains phénomènes commerciaux se rapportant à cette industrie, dont on ignorait à ce jour l'existence. Ces statistiques font découvrir, notamment, l'importance et le dynamisme des comptoirs mis en place outre Atlantique, par les français, dès le début du siècle, aux Etats-Unis, au Brésil, au Pérou, à Cuba, à Porto-Rico, en Guadeloupe, dans l'île de la Réunion, etc. Nul ne soupçonnait, par exemple, que Cuba et Porto-Rico aient été, en 1839 , notre quatrième client après la Belgique, les Etats-Unis et 1'Allemagne, que le Pérou ait occupé le même rang en 1848, et que le Chili ait acheté, en 1841, 12,5\% de notre production, devenant ainsi le troisième de nos clients! Ces chiffres procurent également l'occasion de rectifier certaines erreurs, colportées depuis des générations, en permettant de situer très précisément dans le temps, le début de certains processus commerciaux, comme, par exemple, le déclin du marché extérieur de l'édition française (qui ne remonte pas, comme on le croyait, aux années précédant la première guerre mondiale, mais à celles qui sont antérieures à la guerre de 70).

Les fluctuations de ces statistiques sont également significatives par la manière dont elles accusent les événements politiques ou les 
crises sociales et économiques traversés par le pays. Ainsi, par exemple, la guerre de 1870 avec la Prusse et les autres états allemands se traduit par une chute des ventes vers l'étranger de $34,6 \%{ }^{4}$; la guerre de Sécession provoque une coupure totale de nos exportations vers les Etats-Unis en 1863, alors que ce pays se plaçait au troisième rang de nos clients en achetant entre 14 et $15 \%$ de notre production avant 1861; la politique de libre échange d'union douanière, imposée par Napoléon III, à partir de 1860, engendre une flambée des chiffres d'importations qui passent de $7.007 \mathrm{kgs}$. en 1860 à 55.666 kgs. en 1868 !

Avant de poursuivre plus avant ces observations dans le détail, par l'analyse des chiffres fournis dans chacun des tableaux décennaux, voici quelques précisions sur les documents qui ont servi à notre étude, ainsi que sur la manière dont nous les présentons à l'intérieur de cet article.

En préambule, des réserves de prudence s'imposent quant à la fiabilité à 100\% des données fournies par ces statistiques. En effet, une question se pose: toute musique exportée ou importée faisait-elle l'objet de déclaration auprès de l'administration des douanes? La quantité déclarée était-elle conforme ou inférieure à la réalité? Le contrôle des douanes était-il aussi sévère à l'exportation qu'à l'importation? L.J. Magnan fait remarquer qu'à cette époque, les chiffres déclarés à l'exportation ne témoignaient pas de la même exactitude que ceux qui s'appliquaient aux importations ${ }^{5}$. Pour protéger le commerce français, l'administration des douanes, avait tendance à exercer un contrôle plus sévère sur les opérations d'importations, que sur celles d'exportations. Vraisemblablement les chiffres d'exportation que nous présentons ici, pour être plus conformes à la réalité, devraient être légèrement rehaussés. De plus les musiciens voyageaient beaucoup, surtout dans les pays d'Europe; les échanges entre l'Italie, les pays germaniques, l'Angleterre et la France étaient fréquents, rien n'empêchait les voyageurs de charger leurs malles de musique (de nombreuses correspondances de compositeurs ou d'éditeurs en font état), mais il est peu probable que ces transports individuels, s'ils 
avaient été pris en compte, aient modifié de manière notable les résultats que divulguent les registres des douanes. Pour contrôler la justesse des chiffres se rapportant à la musique, il aurait fallu pouvoir les comparer à d'autres, issus de sources différentes. Malheureusement, jusqu'ici, nous n'avons pas trouvé de données comparables. Les registres de dépôt légal sont dans ce domaine de peu d'utilité, puisque les dépôts d'ouvrages publiés par les éditeurs français ont été effectués de manière relativement fragmentaire, même au cours des dernières dévernies du XIXème siècle ${ }^{6}$. Malgré les doutes que nous venons d'évoquer sur l' exactitude des statistiques douanières, force est de constater la finesse des écarts de chiffres enregistrés d'une année sur l'autre, pour un même pays, ce qui tendrait à démontrer que ces enregistrements ont quand même été effectués avec une certaine rigueur. Quoiqu'il en soit, dans leur détail, elles devront être observées avec prudence, car elles peuvent comporter une certaine marge d'inexactitude; en revanche, dans leur globalité, ces statistiques semblent fiables et elles reflètent indubitablement les grandes lignes de l'évolution des échanges commerciaux dans le domaine de la musique.

Deux grandes rubriques composent ces tableaux décennaux: les importations (avec les "quantités arrivées" et les "quantités mises en consommation"7 et les exportations (avec "quantités exportées, marchandises françaises"). La musique s'y trouve classée sous le "commerce spécial", dans la catégorie "papier et ses applications". Sous une rubrique intitulée "pays de provenance", ou "pays de destination" sont énumérés les noms des pays dont le commerce est détaillé année par année; ces pays diffèrent d'une décennie à l'autre et ils sont beaucoup moins nombreux dans la rubrique "importations" que dans celle des "exportations". Malheureusement, plus on avance dans le siècle, moins ces pays sont détaillés dans l'une ou l'autre de ces rubriques, ils sont alors regroupés sous un chapeau générique intitulé "autres pays", ou "tous pays confondus", ce qui interrompt les possibilités de confrontation pour certaines périodes. En regard de chacun des pays cités figurent, à l'intérieur d' une colonne annuelle, les quantités (en kilos) ayant fait l'objet 
des échanges. Un total annuel est établi au bas de la colonne avec son équivalent en monnaie ${ }^{8}$.

Compte tenu de la difficulté d'accès à ces tableaux, nous avons choisi d'insérer dans cet article la totalité des statistiques qu'ils contiennent, de manière à permettre à chacun d'y puiser les informations qui pourraient lui être utiles. Nous avons ajouté à ces données trois éléments: $1^{\circ}$ ) le pourcentage que représente chacune des quantités échangées, calculé en fonction du total annuel des échanges, $2^{\circ}$ ) un classement annuel des pays, selon 1' ordre d'importance des échanges enregistrés, $3^{\circ}$ ) des graphiques, sous forme d'histogrammes ou de secteurs, qui regroupent d'une manière plus parlante à l'oeil, une partie des chiffres présentés dans les tableaux. De plus, à la fin des tableaux décennaux, nous avons totalisé les résultats annuels de chaque pays, de manière à pouvoir les classer en fonction de leur moyenne décennale.

\section{Importations-exportations}

\section{1) Examen comparatif global}

Lorsqu'on examine globalement les mouvements des échanges de musique entre 1827 et 1896, on est d'abord surpris par la manière dissemblable et opposée dont ont évoluées les importations et les exportations au cours de la même période (cf. planches 1,2,3).

Alors que les ventes de musique française vers l'étranger se traduisent par une progression continue et régulière (planche 2), qui correspond à la courbe générale de l'ensemble des exportations françaises ${ }^{9}$, la pénétration des éditions étrangères, après avoir stagné pendant plus de trente ans, s'effectue de manière explosive et brutale, avec une répartition de $93 \%$ de la totalité des importations entre 1867 et 1896, dont $46 \%$ entre 1887 et 1896 (planche 3). La cause essentielle de la disparité constatée entre importations et exportations est d'abord d'origine politique. Il faut rappeler que jusqu'en 1860 la politique douanière de la France était inscrite dans un climat hautement protectionniste. Les droits d'entrée, 
extrêmement élevés pour la musique, qui s'échelonnaient entre 12 et $29 \%$ de la valeur déclarée des quantités entrées, (entre 1840 et $1850)^{10}$ décourageaient les acquéreurs éventuels d'éditions étrangères. De plus, le commerce français de l'édition musicale poursuivait, depuis la fin du XVIIIème siècle, sa phase ascensionnelle, avec des éditeurs extrêmement productifs (Richault, Schlesinger, etc.) et compétitifs (collections d'éditions "à bon marché" créées par Schlesinger, Latte, Launer, Beauvais etc, $)^{11}$. Le répertoire publié couvrait tous les genres de musique: des éditions des grands classiques (intégrales de musique de chambre, de symphonies et concertos d'oeuvres de Mozart, Haydn, Beethoven, publiés par Sieber, Imbault, Schlesinger, etc.), aux oeuvres de musique légère (arrangements d'airs à la mode, danses pour les bals), en passant par les oeuvres lyriques et le répertoire pour piano, extrêmement varié, qui répondait à la vogue de cet instrument. Après 1860, une autre dynamique commerciale prend corps. La politique plus libérale de l'Empereur Napoléon III et les séries de mesures économiques qu'il fait adopter pour favoriser le développement de l'industrie, notamment la réduction des taxes d'entrée des marchandises en France ${ }^{12}$, modifient complètement les habitudes commerciales. Aussitôt que les traités de commerce sont signés avec l'Angleterre (1860), la Belgique (1861), le Zollverein (1862), l'Italie (1863), la Suisse (1864), la Suède et la Norvège (1865), les Pays-Bas (1865), l'Espagne (1865), le Portugal (1866), l'Autriche (1866) ${ }^{13}$, les effets s'en font sentir, mais pas dans le sens attendu pour le commerce de l'édition musicale. Les entrées massives d'éditions étrangères font basculer l'équilibre commercial de cette industrie. De plus, la compétitivité des éditions en provenance des pays de l" Association allemande", tant sur le plan des prix, du fait de la modernisation des techniques d'impression utilisées par des maisons telles que Breitkopf, Schott, Litolff, Simrock, Peters, etc., (presses mécaniques, utilisation des machines à vapeur, moteurs à gaz, reliure industrielle), que de l'ampleur du répertoire publié, réorienté en fonction des nouvelles demandes des amateurs (grandes éditions critiques, collections bon marché 
des classiques et des contemporains), provoque le ras de marée qui apparaît de manière si criante sur les graphiques des planches 1 et 3 , puisqu'à elles seules, les éditions allemandes représentent, entre 1860 et 1896, 73,5\% des importations totales entrées en France ${ }^{14}$ !

\section{2) Exportations}

La courbe de croissance des exportations de musique entre 1827 et 1896 suit le rythme de croissance général du commerce, elle triple pratiquement sur soixante dix ans (si l'on tient compte du fait que les chiffres des exportations sont un peu en deça de la réalité $)^{15}$. On est tenté de comparer ce résultat au rythme d'accroissement du dépôt légal sur la même période (malgré toutes les réserves que nous avons émises sur la manière dont était effectué ce dépôt par les éditeurs). Cette opération fait apparaître une progression par sept, ce qui tendrait à démontrer, en supposant que les chiffres du dépôt légal n'aient pas été trop éloignés de la réalité, qu'un tiers de notre production nationa'e aurait été destinée à l'exportation. Pendant trente ans, entre 1827 et 1857, la moyenne des exportations de musique est demeurée sept fois supérieure à celle des importations ${ }^{16}$. L'écart s'amoindrit ensuite, en tombant à 4, mais reste à ce niveau jusqu'en 1862. C'est seulement à partir de 1865-1866 (années au cours desqueiles nous avons acheté entre deux fois et demi et cinq fois plus de musique aux pays de $1^{\prime}$ Association allemande ${ }^{17}$ que nous ne leur en avons vendu ${ }^{18}$ ), que le commerce de l'édition française commence à ne plus pouvoir faire face à la concurrence étrangère. Moins dynamiqu* qu'il n'était dans la première moitié du siècle, insuffisamment adapté sur le plan de l'outillage, pas assez compétitif au niveau des prix (faute justement de ne pas avoir su s'adapter aux innovations techniques d'impression et de fabrication), répondant moins aux besoins des consommateurs, il amorce sa periode de déclin. Cette tendance se confirme dès 1867 , lorsque pour la première fois nos exportations sont inférieures aux importations $(36.400 \mathrm{kgs}$. achetés contre 31.169 kgs.vendus). L'écart se creusera ensuite en s'accélérant. En dépit 
des tentatives entreprises dès 1866 , et pendant la guerre de 1914, par des groupes d'éditeurs français, liés dans des organismes de défense (Dufour, Brandus Colombier; Heugel, Leduc, Lemoine, Fromont, etc.), pour lutter et juguler "les ravages" causés par l'invasion des éditions allemandes, rien ne permettra d'enrayer ce phénomène fatal à l'édition musicale française ${ }^{19}$.

\section{3) Importations}

Si l'on observe à présent la courbe générale des importations de musique, on est étonné de constater la résistance du marché français aux éditions étrangères et la durée de ce blocage, qui tient jusque dans les années 1860. Pendant quarante ans, la constance des chiffres enregistrés dans les statistiques est surprenante: sur la totalité des importations enregistrées entre 1827 et 1896, seulement une proportion de $0,95 \%, 0,95 \%, 1,03 \%, 4 \%$ pénètrent sur notre sol entre 1827 et $1866^{20}$ (cf. planche 3). Entre 1827 et 1862 la moyenne annuelle des importations est de six fois inférieure à celle des exportations. Sur cette période les chiffres d'importations s'échelonnent entre 506 et 9.647 kgs., alors que ceux des exportations varient de 10.550 et $26.184 \mathrm{kgs}$. A l'inverse, après 1862 et jusqu'en 1896, c'est le chiffre des importations qui dépasse celui des exportations, en le doublant $(2.580 .753 \mathrm{kgs}$. importés contre $1.231 .405 \mathrm{kgs}$. exportés). La politique protectionniste de la France n'explique pas à elle seule ce rejet pour les éditions étrangères, constaté durant cette première moitié du siècle. L'hypothèse que nous avons émise précédemment semble plus vraisemblable : notre production couvrant pratiquement tous les genres de musique, la majorité des grands auteurs étrangers étant publiés aussi en France (les italiens chez Pacini, Troupenas, Latte, les allemands chez Schlesinger, la musique instrumentale chez Richault, etc.), l'achat à l'étranger s'avérait relativement superflu.

Après 1856, l'augmentation des importations dans notre pays a proportionnellement été beaucoup plus importante que ne l'a été celle de nos exportations vers l'étranger. Pour juger de l'importance 
de ces progressions, comparons la moyenne des quantités importées en France entre 1857 et 1866 à celle de la période 1887-1896 : on constate une progression de $91,3 \%$ si l'on inclut l'Allemagne, et de $80 \%$ sans l'Allemagne. Si l'on fait de même avec les exportations au cours des mêmes périodes on n'obtient qu'une augmentation de $22,6 \%{ }^{21}$. L'afflux soudain d'éditions allemandes ${ }^{22}$ sur le marché français, n'est donc pas l'unique cause du déséquilibre de la balance commerciale de l'industrie de l'édition musicale. L'active politique éditoriale menée par certains pays européens (Autriche, Belgique, Angleterre) a également contribué à entraîner la France dans sa chute. Nos échanges avec la Belgique et l'Angleterre en fournissent des exemples concrets: jusqu'en 1866 on vendait presque neuf fois plus de musique à la Belgique qu'on ne lui en achetait, cette proportion tombe à une fois et demie entre 1877 et 1896 , bien que le chiffre de nos exportations vers ce pays ait continué à croître en passant de 4.307 kilos en 1837 à 28.100 kilos en 1895 ; en Angleterre, nos exportations dépassaient de vingt-quatre fois nos importations, entre 1837 et 1856 , elles se sont réduites à une fois et demie entre 1877 et 1886 , alors que nous continuions à augmenter nos exportations vers ce pays : 1.715 kilos en 1837 , et 9.565 kilos en 1896 23. Toutefois, si le boom germanique n'avait pas eu lieu, le désastre aurait été moindre.

Une autre expérience nous a également intéressée: celle de comparer le chiffre le plus bas des exportations $(10.550 \mathrm{kgs}$. en 1831) au chiffre le plus élevé ( $58.095 \mathrm{kgs}$. en 1895), elle fait apparaître une progression de 5,5; si l'on procède de même avec les importations (506 kgs. en 1838 et $181.031 \mathrm{kgs}$. en 1895), on trouve un chiffre multiplié par 357 !

Les résultats des statistiques d'importations "quantités arrivées" (chiffres sur lesquels nous sommes fondés), ne prennent toute leur signification que s'ils sont comparés à ceux des "quantités mises en consommation". Des écarts parfois importants les éloignent et modifient sensiblement le parallèle qui peut être établi entre exportations et importations. Qu'entendait l'administration des douanes par "mises en consommation"? Nous supposons qu'il devait 
s'agir des quantités effectivement vendues, après un délai d'un an, puisque les taxes d'entrées étaient prélevées à la fin de chaque année sur ces "quantités mises en consommation". Toutes les quantités arrivées étaient loin d'être consommées, surtout dans les premières décennies que nous étudions. Le tableau ci-dessous le fait clairement apparaître :

\section{IMPORTATIONS : \\ QUANTITES ARRIVEES ET CONSOMMEES}

années

\section{Moyennes décennales} arrivage consommation $\%$ décennal de la consommation

$\begin{array}{lrrr}1837-1846 & 2.558 & 426 & 16,65 \% \\ 1847-1856 & 2.789 & 642 & 23,02 \% \\ 1857-1866 & 10.805 & 6.332 & 58,60 \% \\ 1867-1876 & 49.634 & 43.421 & 87,48 \% \\ 1877-1886 & 77.664 & 67.489 & 86,90 \% \\ 1887-1896 & 124.298 & 111.904 & 90,03 \%\end{array}$

Comme on le voit, ces chiffres renforcent ce que nous avons constaté précédemment, à savoir la faible consommation d'éditions étrangères par les français tout au long de la première moitié du XIXème siècle: successivement $16,6 \%, 23 \%$, et $58 \%$ des quantités arrivées, entre 1837 et $1866^{24}$. Ensuite l'écart s'amenuise, la "consommation" avoisine les $90 \%$ des quantités arrivées entre 1867 et 1896. Lorsque l'on regarde dans le détail la "consommation" par contrées, l'on constate que certains pays ont su évaluer avec plus de justesse que d'autres, les quantités nécessaires aux besoins de la France. Les italiens, par exemple, les ont surévaluées. De plus, ils semblent n'avoir jamais pris conscience de la disproportion de leurs envois par rapport aux achats des français ${ }^{25}$. Entre 1837 et 1896 , successivement $7,8 \%, 3 \%, 14 \%, 21 \%, 20 \%$ et $40 \%$ des quantités arrivées d'Italie ont été "consommées"! Même à la fin du 
siècle, alors que la "consommation" générale représentait 90\% des quantités importées arrivées, les éditeurs italiens surévaluaient leurs envois de $60 \%{ }^{26}$.

Les belges ont fait la même erreur que les italiens, mais sur un laps de temps plus court. Entre 1837-1846, seulement 4,5\% de leurs envois ont été consommés par les français, en revanche, au cours des décennies suivantes, l'écart s'est amenuisé pour rejoindre : $73,7 \%, 90,2 \%, 98,8 \%$ et $95 \%{ }^{27}$.

L'Angleterre a su moins bien que la Belgique ajuster ses envois vers la France: sur les quantités proposées entre 1837 et 1856, puis entre 1876 et 1886 , seulement $40,4 \%, 56 \%$ et $42 \%$ ont été mises en consommation.

Les pays germaniques ont également mis quelques décennies avant d'équilibrer leurs quantités : entre 1837 et $1846,35,8 \%$ des ouvrages importés ont trouvé consommateurs, pour les décennies suivantes la consommation est passée successivement à $62 \%$, $88,6 \%, 98 \%, 95 \%$ et $99 \%$

Quant aux pays englobés sous la rubrique "autres pays" 28 , eux aussi ont eu des difficultés à doser leurs envois en fonction de nos besoins: successivement $6,66 \%, 14,17 \%, 54,28 \%, 78,13 \%, 55,8 \%$, $54 \%$ des quantités reçues entre 1837 et 1896 ont été consommées. Pour clore ce paragraphe, signalons encore quelques contrastes ponctuels frappants: un arrivage en 1841 de $2392 \mathrm{kgs}$. de musique en provenance des Pays-Bas pour une "consommation" de 5 kgs.; un autre en 1837, de $1.312 \mathrm{kgs}$. en provenance d'Angleterre pour une utilisation de $490 \mathrm{kgs}$., enfin un dernier de $1.767 \mathrm{kgs}$. en provenance d'Allemagne (en 1860), pour une "mise en consommation" de $434 \mathrm{kgs}$.

Avant d'entreprendre l'observation des statistiques par pays, signalons encore quelques intéressantes fluctuations de chiffres que nous avons notées dans la succession générale des exportations et des importations: la chute de $29,7 \%$ des exportations en 1831 (par rapport aux exportations enregistrées en 1830) suivie de celle de $37 \%$ des importations au cours de la même année; un rapprochement peut être établi entre ces baisses et la crise économique liée à la 
Révolution de Juillet, qui fut suivie d'une épidémie de choléra entre 1831 et 1832 . La crise de $1847-1848^{29}$ n'est certainement pas étrangère non plus à la diminution de $26,6 \%$ de nos exportations entre 1847 et 1848; les effets de cette crise ne s'étant répercutés qu'un an plus tard sur les importations, avec une chute de 56,7\% (3.828 kgs. entrés en 1848, 1.655 kgs. en 1849). Après l'entrée en guerre avec la Prusse, en 1870 , nos ventes à l'étranger se réduisent de $34,6 \%$, ceci affecte peu les chiffres d'importations, qui doublent pratiquement dès 1872 . En 1880, on constate, par rapport à 1879 , une baisse spectaculaire de $66 \%$ de nos exportations, les rapports qu'on entretenait avec l'Allemagne et la Belgique semblent en avoir été responsables, car ces deux pays ont réduit leurs achats de musique française de 99 et $98 \%$.

\section{Pays de destinations et lieux de provenances}

L'observation des mouvements commerciaux par pays, sera fragmentaire, car les pays cités diffèrent d'un registre décennal à l'autre. Les lieux d'exportations ne sont détaillés que pendant trente ans (1827-1856), avec une liste d'une vingtaine de pays. Cette tranche de temps est suffisamment longue cependant pour avoir une vision assez complète de l'évolution du commerce extérieur de la France, au cours de la première moitié du XIXème siècle. Malheureusement, il n'en est pas de même pour la période couvrant la seconde moitié du siècle: la description des lieux de destination se borne à huit, puis trois pays (Belgique, Allemagne, Angleterre), chaque pays étant englobé, au fur et à mesure de sa suppression, sous une rubrique "autres pays". Quant aux lieux de provenance des importations, ils sont encore moins nombreux, même avant 1850. Seuls les échanges avec l'Allemagne ont fait l'objet d'enregistrements continus ${ }^{30}$. Malgré ces interruptions, les informations fournies par ces registres restent précieuses, car elles cernent de manière ponctuelle et quantitative la courbe évolutive de notre commerce extérieur, et celle de nos achats, pendant un laps de temps relativement significatif. 
Ce qui frappe en premier lieu en regardant les tableaux, c'est l'amplitude du champ de diffusion des éditions françaises à l'étranger, notamment Outre-Atlantique, dès 1827. L'esprit d'entreprise dont ont fait preuve les éditeurs parisiens, dès les premières années du XIXème siècle, est remarquable, car rien n'était moins sûr, à cette époque, que d'installer des comptoirs de vente dans des pays aussi éloignés que les îles de l'archipel des Antilles, ou le Brésil, pour répondre aux besoins de musique d'une classe bourgeoise. Récupérer le produit de ventes effectuées dans des contrées aussi reculées n'était pas non plus chose aisée, il fallait de l'audace pour tenter l'aventure. Nous l'avons vu, les éditeurs français en ont eu pendant un temps et en ont retiré des résultats concluants. Le développement des moyens de transports (bateaux réguliers, réseau ferré, routes avec percement de tunnels), qui n'a cessé de s'améliorer tout au long du siècle, a, bien entendu, contribué à la réussite de cette implantation.

\section{1) Pays d'Outre-Atlantique}

Des pays situés outre-Atlantique, les Etats-Unis ont été notre principal client. Une vie musicale était déjà bien établie dans plusieurs villes de ces états au début du XIXème siècle, principalement en Nouvelle Orléans (où la musique française, notamment les opéras de Boieldieu, Adam, étaient très appréciés ${ }^{31}$ ), à Philadelphie, Boston et New-York. Bien que les droits d'entrée aux Etats-Unis aient été assez élevés, les Etats-Unis ont acheté des éditions françaises en quantité importante pendant trente ans. Ils ont occupé la seconde place parmi nos acheteurs entre 1837 et 1856, avec l'acquisition de $11 \%, 7 \%$ et $12, \%$ de nos exportations; ils se sont même classés premiers en 1836 et 1850 avec $23 \%$ et $20 \%$ de nos exportations. Seule la Guerre de Sécession mit un terme cette relation privilégiée, à partir de 1861. Malheureusement, les registres décennaux ne permettent pas de savoir comment s'est effectuée la reprise des ventes après 1867 (les Etats-Unis ayant été regroupés sous la rubrique "tous pays confondus"). Le commerce de l'édition musicale 
comptait, dans ce pays, dès 1820 , une poignée d'éditeurs relativement actifs. Ceux-ci faisaient office de diffuseurs des éditions françaises, comme le révèlent leurs noms cités, au titre de dépositaires, dans les inventaires après décès d'éditeurs français, entre 1827 et 1850 (J. Hewitt à New-York, G. Willig, G E. Blake, Lee \& Walker à Philadelphie, G Graupner et O Ditson à Boston, Laporte à la Nouvelle-Orléans, etc.). D'après Pontécoulant ${ }^{32}, 30$ marchands de musique étaient implantés à Cincinnati en 1851 !

La vie musicale dans les Antilles, à Cuba, à Haïti, à PortoRico, aux Iles Vierges (Saint Thomas), ou en Guadeloupe, ne nous laissait pas supposer qu'elle ait offert de tels débouchés de vente aux éditeurs de musique français entre 1827 et 1856; une moyenne d'environ $5 \%$ de nos exportations a été pendant trente ans destinée à l'ensemble de ces îles, ce qui les plaçait au rang de cinquième client de la France.

Le commerce de la France avec le Chili se distingue par l'achat d'une quantité de musique curieusement élevée, effectuée en 1841: $3.285 \mathrm{kgs}$, soit $14 \%$ de nos exportations, alors que la moyenne des ventes qui lui étaient destinées oscillaient entre 1 et $4 \%$.

Le Brésil reste constant dans sa moyenne d'achats avec notre pays en acquérant $3,5 \%$ de nos exportations. Les seules augmentations notables se situent en 1848 et 1849 , années au cours desquelles 5 et $7,8 \%$ de nos exportations leurs ont été destinées, ce qui l'a placé au rang de troisième acheteur en $1849^{33}$.

Le commerce avec l'Argentine (Rio de Plata) n'a commencé à se développer qu'au cours de la décennie 1847-1856, avec une moyenne de 3,1\%. Seule l'année 1855 fait apparaître un achat important de 3.883 kilos (soit $12,5 \%$ de nos exportations), classant Rio de la Plata comme notre troisième principal client. Une percée de son voisin, l'Uruguay, est à noter entre 1848 et 1851 , mais les ventes avec ce pays sont restées très faibles (moyenne située autour de $0,3 \%$ avec une poussée de $2,17 \%$ en 1848).

On constate, la même année, en 1848, une augmentation identique de nos exportations vers le Pérou, alors que celles-ci se 
situaient autour de $1,3 \%$ (pour la décennie 1847-1856), elles passent à $6,23 \%$ en 1848 .

Les ventes avec le Mexique attestent d'une croissance régulière au cours des décennies 1827-1856, en passant de $1,2 \%$ à $2,4 \%$. Un achat plus important de $1.262 \mathrm{kgs}$., soit 7,8\% de nos exportations, rompt ce rythme en 1843, et lui confère le rang de cinquième client de la France.

Si l'on se tourne à présent vers l'Océan indien, l'on constate également l'existence d'un marché: à la Réunion (Bourbon) et dans l'île Maurice. Loin d'être comparable à celui des Antilles, ce marché n'était pas négligeable, surtout dans les premières années du XIXème, où l'île de la Réunion absorbait jusqu'à 6,7\% (en 1829) de nos exportations. Il décroît ensuite à $0,9 \%$ au cours de la décennie 1847-1856. Les ventes en île Maurice, suivent la courbe inverse en progressant de 1 à $\%$, entre 1827 et 1856 .

\section{2) Pays d'Europe}

Entre 1827 et 1856, deux tiers des exportations françaises d'éditions musicales ont été destinées aux pays d'Europe, soit 70\% de la totalité de nos exportations (dans ce pourcentage sont inclues les quantités apparaissant sous la rubrique "autres pays", représentant entre 3 et $6 \%$ ). Après 1856, la liste des pays ayant été réduite, il est impossible de connaître les quantités exportées en Europe et dans le reste du monde.

Au cours des soixante-dix années que couvre cette étude, la Belgique a été notre principal et meilleur client. Bien qu'ayant eu elle-même une activité éditoriale importante au cours du XIXème siècle (dans des villes telles que Bruxelles, Liège, Anvers et Gand ${ }^{34}$ ), elle n'a cessé de s'approvisionner en éditions françaises, en achetant en moyenne $33 \%$ de nos exportations, occupant ainsi le rang de premier client de France. Ses achats ont été en constante progression, quintuplant en soixante dix ans. Son tarif douanier, très libéral, a favorisé les entrées de musique étrangère sur son territoire. Les quantités qui lui ont été fournies par la France ont 
oscillées entre $19,6 \%$ et $41,7 \%$. Seule ombre à ces achats : une commande de $241 \mathrm{kgs}$. en 1880 , qui provoque une chute de $98 \%$ par rapport à la quantité de musique française acquise l'année précédente $(15.710 \mathrm{kgs}$.).

En retour, les éditeurs belges nous ont envoyé leurs éditions, d'abord en quantités assez modestes, puis avec une nette progression à partir de 1857, passant de 3.414 kgs. (entre 1847-1856) à 122.902 kgs. (entre 1886 et 1896), soit une augmentation chiffrée par trente $\operatorname{six}^{35}$. Cette progression, particulièrement sensible à partir de 1862 et qui n'a cessée de croître ensuite, est vraisemblablement liée à l'installation d'une succursale belge à Paris, en 1861. De France, le directeur de cet établissement belge, Jean-Baptiste Katto ${ }^{36}$, était mieux placé pour contrôler la diffusion et assurer la promotion des ouvrages publiés en collaboration avec la maison de Bruxelles.

Se partagent ensuite les rôles de meilleurs clients de la France: l'Angleterre et le conglomérat de pays regroupés sous "autres pays". Les quantités exportées vers l'Angleterre ont été en moyenne trois fois inférieures à celles exportées vers la Belgique. Malgré cette importante différence, l'Angleterre achetait en moyenne plus de $12 \%$ de nos exportations. Ses achats ont triplés sur soixante-dix ans. Le traité signé en 1860 entre la France et l'Angleterre n'a pas provoqué d'ascension spectaculaire des achats ou des ventes d'un pays vers l'autre. On note, sans pouvoir l'expliquer, une chute importante des achats de l'Angleterre en 1864.

L'évolution de la courbe des importations d'éditions musicales anglaises en France sera fragmentaire, compte tenu des lacunes relevées dans les registres décennaux. Les seules indications précises que nous ayons portent sur la période située entre 1837 et 1856 et 1877 et $1886^{37}$. Ces importations se sont cantonnées à de faibles quantités, tournant autour d'une moyenne de $5 \%$. Une progression notable des quantités entrées en France est à signaler entre la décennie 1847-1856 (1.219 kgs.) et celle de 1877-1886 (27.406 kgs.), ainsi qu'une chute de nos achats en 1885 (924 kgs.).

Avant d'observer les statistiques réunies sous la rubrique "autres pays", il est nécessaire de préciser que cette rubrique ne 
regroupe pas les mêmes pays au fil des années et selon qu'il s'agit d'importations ou d'exportations. Entre 1857 et 1896, elle englobe un nombre beaucoup plus vaste de contrées pour les exportations, qu'elle n'en réunit pour les importations, car peu de pays dans le monde avaient une activité éditoriale de musique (une fois retirés les pays régulièrement cités dans les statistiques: Allemagne, Belgique, Angleterre, Italie et Suisse). Seuls des pays comme l'Autriche (avec Haslinger, Diabelli ou Mechetti à Vienne), l'Espagne, la Russie (avec Gutheil, Jurgenson, Bessel à Moscou ou Saint Petersbourg), les Pays-Bas (Weygandt à la Haye, Plattner à Rotterdam), les Etats-Unis étaient susceptibles de nous vendre leurs éditions.

Jusqu'en 1856 les renseignements fournis par la rubrique "autres pays", dans le chapitre "exportations", n'offrent qu'un intérêt relatif, puisque les envois de la France vers l'étranger sont détaillés avec plus d'une vingtaine de pays. Les quantités qui restent sous cette rubrique oscillent entre 2 et $6 \%$. En revanche après 1856 , les chiffres d'exportations destinés aux "autres pays" sont beaucoup plus révélateurs, car ils représentent un tiers de la totalité des exportations générales, les deux autres tiers ayant été répartis entre la Belgique, l'Allemagne, l'Angleterre ou la Suisse

Les statistiques "autres pays" portant, à partir de 1857, sur les importations confirment le faible pourcentage d'achats d'éditions étrangères effectués par la France, hormis les acquisitions allemandes, et loin derrière, anglaises, italiennes, belges ou suisses. Seulement 3 à $8 \%$ de nos achats provenaient de ces autres pays indéterminés.

Si l'évolution du commerce de la musique entre la France et l'Allemagne est particulièrement significative après 1863 , la courbe des échanges effectués entre 1827 et 1856 l'est également, car elle fait apparaître la manière dont a évolué notre commerce avec ce pays avant qu'il ne connaisse un essor spectaculaire.

L'on constate tout d'abord qu'au cours de la première moitié du XIXème siècle l'Allemagne n'a jamais été un gros acheteur d'éditions françaises. Ses achats ont même été en constante 
régression de 1827 à $1856^{38}$; malgré cela, les quantités vendues à ce pays sont restées supérieures de deux fois à celles qui ont pénétrées en France jusqu'en 1856. De 1837 à 1856 les importations de musique allemande n'ont représenté que 44,4\% du chiffre des exportations et les français n'ont "consommé" que la moitié des éditions allemandes entrées dans le pays ${ }^{39}$. Ce n'est qu'au cours de la décennie suivante que la balance entre nos échanges a basculé. Entre 1857 et 1866, les importations ont dépassé les exportations de $26,4 \%$ et la "consommation" des français en éditions allemandes a augmenté également: $88 \%$ au lieu de 44\% des quantités entrées sur notre territoire ont été achetées ${ }^{40}$. Entre 1877 et 1896 (interruption des statistiques d'exportations entre 1867 et 1877) les exportations n'ont plus représenté que $6,4 \%$ des quantités importées; sur le million et demi de kilogrammes de musique allemande entré en France au cours de ces vingt années, la quasi totalité $(97,8 \%)$ a été consommée ${ }^{41}$ ! Au cours de la dernière décennie, la vogue du wagnérisme en France ne fut certainement pas étrangère non plus à l'augmentation de "consommation" d'éditions germaniques par les français.

La soudaineté avec laquelle les éditions allemandes ont envahi le marché français après 1863, n'est pas uniquement liée à l'abaissement des droits de douanes et à la compétitivité des prix allemands. Cette prise de possession avait été préparée de longue date par les éditeurs des pays germaniques. Très tôt dans le siècle ceux-ci avaient pris conscience des débouchés commerciaux que pouvait représenter le marché français. Dès les années 1830, ils avaient commencé à poser des jalons dans la capitale française en y ouvrant des succursales ou en y choisissant des dépositaires. En 1822, Adolphe-Martin Schlesinger de Berlin avait chargé son fils, Maurice, d'assurer le dépôt de ses publications; en 1826 la maison Schott de Mayence avait ouvert une filiale parisienne, avec à sa tête Jean et André Schott. Elle avait ensuite confié, à partir de 1830 , la représentation exclusive de ses éditions aux dépositaires français Claude Heu (entre 1830 et 1833) et Jean Hartmann (entre 1838 et 1857), rémunérés au pourcentage sur les ventes et par des 
commissions sur les droits d'auteurs. En 1838, Simrock de Bonn avait mis en dépôt ses publications chez Frey. Litolff de Brunswick avait fait de même d'abord avec Félix Janet jusqu'en 1865, puis avec Enoch. En 1862, la maison Schott de Mayence avait ouvert, pour la seconde fois, une filiale parisienne. Breitkopf de Leipzig, dont les éditions étaient si compétitives sur le plan des prix, avec leurs collections à très bon marché des musiciens classiques, avait préféré choisir la formule du dépositaire plutôt que celle de la filiale. C'est à Durdilly d'abord, puis à Costallat, qu'elle confia le soin d'être leurs représentants généraux et exclusifs. Peters de Leipzig fit de même avec l'éditeur Auguste Durand. Tous ces exemples illustrent combien les éditeurs allemands étaient déjà bien implantés à Paris avant même la guerre de 1870 et combien le terrain était bien préparé pour la conquête du marché français. La politique de libre échange imposée par Napoléon III ne fut plus que l'étincelle qui donna le coup d'envoi.

Quelques transactions vers l'étranger valent encore la peine d'être relevées, notamment nos envois vers la Russie. Consignés entre 1827 et 1856, leur progression est intéressante car les quantités envoyées à ce pays doublent d'une décennie à l'autre: $2.077 \mathrm{kgs}$., puis $4.140 \mathrm{kgs}$., enfin $8.959 \mathrm{kgs}$. L' installation de l' éditeur français Brandus à Saint-Petersbourg, en 1852, et celle, avant lui, de son associé Dufour, ne sont probablement pas étrangères à l'accueil fait aux éditions françaises. L'ambition de Brandus n'était-elle pas de conquérir ce marché, exploité, selon lui, exclusivement par les éditeurs allemands? Cette conquête fut brève. Si elle fut couronnée de succès en 1852 et 1853, années au cours desquelles la Russie devint le second puis le quatrième client de la France par des achats de $3.327 \mathrm{kgs}$. et $1.909 \mathrm{kgs}$. de musique, soit $13,15 \%$ et $7 \%$ des exportations françaises, la guerre de Crimée y mit un terme: elle occasionna la coupure des expéditions de musique en 1854 et 1855 et la vente du fonds Brandus de Saint-Petersbourg en avril 1855. Le retrait de la Russie des registres de statistiques à partir de 1857 empêche de savoir si la légère reprise des exportations constatée en 1856 s'est poursuivie. 
Nos exportations vers l'Italie, c'est à dire vers le royaume des Deux Siciles, les pays Sardes et la Toscane ont été non seulement peu nombreuses entre 1827 et 1856 mais également en régression en passant de $3,4 \%$ à $1,6 \%$. Les statistiques sont muettes sur les quantités exportées après l'unification italienne. Si les italiens ont fait peu de cas des éditions françaises, les français ont fait de même avec les leurs, car malgré les énormes quantités qui ont pénétrées en France (entre 1837 et 1856 le chiffre des importations dépassait de deux fois celui des exportations ${ }^{42}$ ), ils en ont "consommé" bien en dessous du quart (entre $2,9 \%$ et $21 \%$ jusqu'en 1886 et $40 \%$ entre 1887 et 1896). Le succès de l'opéra italien en France n'a modifié en rien les achats des français qui préféraient acquérir les oeuvres de Rossini, Bellini, Verdi, etc. dans des éditions françaises (avec des traductions françaises des textes) plutôt que dans des éditions italiennes. L'ouverture d'une filiale Ricordi à Paris, en 1888, peut expliquer l'augmentation de $55 \%$ des importations de musique italienne entre les décennies 1877-1886 et 1887-1896.

Les exportations vers la Suisse ont été relativement régulières entre 1827 et 1876 , tournant autour de la même quantité de $9.000 \mathrm{kgs}$. en moyenne, soit entre $3 \%$ et $6 \%$ du chiffre de nos exportations. Les importations en provenance de Suisse se distinguent par une entrée importante de $21.000 \mathrm{kgs}$. au cours de la décennie 1867-1876, soit dix fois plus que nous n'en avions reçu entre 1847 et 1856.

L'Espagne a également offert un débouché intéressant aux éditions françaises, les exportations vers ce pays ont cru régulièrement, à une moyenne de $32,7 \%$ entre 1827 et 1866 . Le Portugal, en revanche n'a été qu'un très médiocre client : $0,8 \%$, $0,5 \%, 2 \%$ de nos exportations lui ont été destinées.

En 1838, deux pays nouveaux font leur apparition dans les registres des douanes, la Turquie et l'Algérie, ils y restent sept ans. Pour peu significatives que soient les quantités expédiées dans ces pays, elles font apparaître néanmoins, la part tenue par la musique européenne dans la vie musicale de ces deux pays. On reste perplexe sur l'achat effectué en 1846 par l'Algérie de $1.362 \mathrm{kgs}$. de musique, qui plaçait ce pays au rang de 3 ème client! On ne sait si cet achat 
fut occasionnel, ou suivi d'autres, faute de suivi dans les tableaux décennaux.

Il est clair que de telles statistiques sont loin de répondre à toutes les interrogations. On aimerait surtout avoir une répartition de tous ces chiffres par catégories musicales: arrangements d'airs d'opéras célèbres, pièces pour piano, recueils pédagogiques d'exercices ou d'études, voire musique de danse ou de bal. Mais d'autres questions surgissent encore, concernant les prix et leur variation, l'influence des règlements douaniers, l'existence et le dynamisme des marchands et dépositaires, etc. Un large champ d'études est ouvert dans tous ces domaines, pour lequel s'impose une collaboration internationale.

\section{NOTAS}

* Artigo publicado na Revue de Musicologie, Paris, Société Française de Musicologie, T. 79, 2:263-296, 1993.

1. Malou HAINE, Les facteurs d'instruments de musique à Paris au $19 e$ siècle, Bruxelles, éditions de l'Université, 1985, s'est aussi appuyée sur les registres des douanes pour établir son étude.

2. Pour les exportations enregistrées au cours de la décennie 1827-1836, la musique n'a pas de rubrique spécifique, elle est inclue avec les gravures et lithographies.

3. Le tableau ci-dessous présente l'estimation en monnaie du kilo de musique, à l'exportation ou à l'importation, entre 1837 et 1896 (la décennie 27-36 est exclue de ce rapport, pour la raison invoquée en note 2). Il met en évidence l'impossibilité de se référer à la monnaie pour effectuer une étude comparative et évolutive du commerce de la musique, faute de base fixe. Les prix étant trop liés aux fluctuations économiques. De plus l'estimation des douanes diffère selon la catégoric, les prix d'éditions importées étant plus élevés que ceux des musiques exportées! On ignore sur quels critères se basaient les douaniers pour établir le prix du kilo de musique.

Prix du kg. de mus. exp.

Prix du kg. de mus. imp.

$\begin{array}{llll}1837-1860 & 12 \mathrm{f} . & 1837-1846 & 27 \mathrm{f} . \\ 1861-1867 & 10,2 \mathrm{f} . & 1847-1866 & 12 \mathrm{f} . \\ 1868-1872 & 8,2 \mathrm{f} . & 1867 & 10,2 \mathrm{f} .\end{array}$




$\begin{array}{llll}1873-1890 & 8 \mathrm{f} . & 1868-1890 & 8 \mathrm{f} . \\ 1891-1893 & 8,1 \mathrm{f} . & 1891-1893 & 7,2 \mathrm{f} . \\ 1894-1895 & 7,3 \mathrm{f} . & 1894-1895 & 6,5 \mathrm{f} . \\ 1896 & 6,4 & 1896 & 5,5 \mathrm{f} .\end{array}$

4. En $1869: 42.740 \mathrm{kgs}$. cxportés, en $1870: 27.946 \mathrm{kgs}$.

5. L.J. Magnan, "Les méthodes d'établissement des statistiques du commerce extérieur en France", dans Revue d'économie politique, 39ème année, 1925 , p. 1189

6. On sait que ceux-ci ne déposaient pas toute leur production, notamment les partitions d'orchestre d'opéras, qui leur coûtaient très cher; cette lacune est regrettable car si toutes les éditions publiées en France avaient été consignées dans ces registres, il aurait été possible d'évaluer de manière précise le pourcentage que représentaient les exportations par rapport à la production générale et ainsi connaître la "consommation" nationale de musique.

7. Les chiffres présentés ici sont ceux des "quantités arrivées" mais nous établirons un parallèle entre ceux-ci et ceux "mis en consommation".

8. Dans un souci d'allègement des tableaux reproduits, nous n'avons pas cru nécessaire d'indiquer, après chaque année, le montant total annuel des sommes, correspondant aux quantités échangées, compte tenu des raisons invoquées et du fait que nous avons présenté, en note 3 , les fluctuations du prix du kilo de musique selon les estimations douanières faites entre 1827 et 1896.

9. R.E. Cameron, "Profit, croissance et stagnation en France au XIXème siècle", dans Economie appliquée, avril-septembre, 1957, t. X, n² 2-3.

10. Ces droits étaient différents selon les marchandises qui pénétraient en France, en 1847, par exemple, 7\% étaient réclamés par les douanes sur les gravures et lithographies, 9\% taxaient les cartes de géographie étrangères et $27 \%$ s'appliquaient à la musique.

11. Cf. A. Devriès et F. Lesure, Dictionnaire des éditeurs de musique français Vol. II : de 1820 à 1914, Genève, Minkoff, 1988.

12. En 1860 les taxes sur les importations de musique sont encore de $10 \%$, en 1862 elles sont réduites à $4 \%$, en 1864 à $2,5 \%$, en 1865 à $0,7 \%$, puis supprimées jusqu'en 1888. En 1892 un nouveau tarif douanier protocteur est voté (cf. A Algoud, Histoire des droits de douane (de leur origine romaine à 1939) et de la politique commerciale de la France, Paris, 1978.

13. Tarif officiel des douanes de France (année 1877), Paris, 1877.

P. Bairoch, Commerce extérieur et développement économique de l'Europe au XIXème siècle, Moudon, E.H.S.S., 1976 
14. Total des importations entrées en France entre 1860 et $1896: 2.603 .450$ $\mathrm{kgs}$, total des importations austro-allemande sur la même période : $1.913 .877 \mathrm{kgs}$.

15. Pour être exact, la moyenne décennale des exportations enregistrées entre 1887 et 1896 par l'administration des douanes, fait apparaître une croissance de deux fois et demie supérieure à celle constatée entre les années 1827-1837.

16. $541.888 \mathrm{kgs}$. d'exportations, contre $79.070 \mathrm{kgs}$. d'importations enregistrés entre 1827 et 1856.

17. Nous engloberons dorénavant sous le nom Allemagne les régions, duchés et villes (Prusse, villes hanséatiques, royaume de Hanovre, Bavièrc, Wurtemberg, etc.), qui actuellement font partie de ce pays, mais qui au XIXème siècle constituaient des territoires indépendants.

18. 1865,1866 : importations en provenance des pays d'association allemande: $11.132 \mathrm{kgs} ., 15.172 \mathrm{kgs}$.; exportations d'éditions françaises vers les mêmes: 4.520 kgs., 2.684 kgs.

19. Cf. sur ce sujet l'article de Ch. TENROC, "Le problème de l'édition française de musique", dans Courrier musical, 1917.

20. $25.593,25.583,27.894 \mathrm{kgs}$.

21. $108.050 \mathrm{kgs}$. importés entre 1857 et 1866 (Allemagne comprise), ou 66.045 (sans l'Allemagne) et 1.242.985 importés entre 1887 et 1896 (avec l'Allemagne), ou 331.428 (sans l'Allemagne) contre $309.446 \mathrm{kgs}$. exportés entre 1857 et 1866 et $400.275 \mathrm{kgs}$. entre 1887 et 1896.

22. Entre 1863 et 1896 les importations allemandes ont représenté $74 \%$ de la totalité des importations.

23. Belgique

1837-1866

1877-1896

Angleterre

1837-1856

1877-1886
Total Imp.

$27.159 \mathrm{kgs}$

172.545

1.725

27.366
Total exp.

$235.844 \mathrm{kgs}$.

292.009

41.379

40.165

24. Nous rappelons la raison pour laquelle nous n'avons pas cité la décennie 1827-1836: les statistiques 1827-1836 présentent, pour les importations, des résultats qui englobent musique, gravures et lithographies, de ce fait, les chiffres de cette décennie sont trop approximatifs, pour les prendre en compte dans ce cas précis.

25. Sous l'appellation Italie sont comprises les villes et les régions qui actuellement font partie de ce pays mais qui, avant l'Unité italienne, constituaient des territoires indépendants (Etats Sardes, royaume des Deux Siciles, Toscane, etc).

26. $136.316 \mathrm{kgs}$. entrés entre 1887 et 1896 contre $54.771 \mathrm{kgs}$. consommés. Il scrait intéressant de savoir si les quantités non consommées étaient 
renvoyées dans les pays d'origine. Aucune information n'est fournie sur ce point.

27. $6.519 \mathrm{kgs}$. importés contre 290 consommés.

28. Aucune indication n'est donnée sur les noms des pays réunis sous l'appellation "autres pays", vraisemblablement devaient en faire partie :l'Autriche, la Russie, l'Espagne, mais selon les décennies le contenu variait.

29. R.E. Cameron, "Profit, croissance et stagnation en France au XIXème siècle", dans Economie appliquée, avril-septembre, 1957, t.X, n 2-3.

30. Sauf entre 1867 et 1876 , années au cours desquelles les exportations sont regroupées dans la rubrique "autres pays".

31. Le Barbier de Séville de Rossini y était même représenté en français en 1823 !

32. A. de Pontecoulant, Organographie. Essai sur la facture instrumentale. Art, industrie et commerce, Paris, Castel, 1861.

33. Mercedes Reis Pequeno recense plus d'une dizaine de commerçants, dont des éditeurs, s'occupant de commerce de musique à Rio de Janeiro, entre 1820 et 1856: cf. DW Krummel, Guide for dating early published music, New Jersey, Joseph Boonin, 1974.

34. Cf. sur ce sujet l'article de Bernard Huys, "Les imprimeurs de musique à Bruxelles au XIXème siècle", dans Fontes Artis Musicae, 35/3 juilletseptembre 1988.

35. Toutefois, ces $122.902 \mathrm{kgs}$. ne représentaient que $9,8 \%$ de la quantité totale des oeuvres importées dans notre pays.

36. Cf. A. Devriès et F. Lesure, Dictionnaire des éditeurs de musique français Vol. II : de 1820 à 1914, Genève, Minkoff, 1988.

37. On ne peut tenir compte des chiffres enregistrés pour la décennie 18271836, puisqu'ils ne correspondent pas à la réalité : la musique ayant été, pour les importations, groupée avec les gravures et lithographies.

38. $22.717 \mathrm{kgs}$. entre 1827 et $1836,14.817 \mathrm{kgs}$. entre 1837 et $1846,5.656$ kgs. entre 1847 et 1856.

39. Quantités importées entre 1837 et 1856: 9.655 kgs., quantités exportées: 21.743 kgs., quantités "consommées": $4.330 \mathrm{kgs}$.

40. Entre 1857 et 1866: quantités importées: $42.005 \mathrm{kgs}$, quantités consommées $37.254 \mathrm{kgs}$., quantités exportées $30.891 \mathrm{kgs}$.

41. Entre 1877 et 1896 : quantités importées: $1.512 .275 \mathrm{kgs}$., quantités consommées: $1.480 .183 \mathrm{kgs}$., quantités exportées: $93.668 \mathrm{kgs}$.

42. $19.512 \mathrm{kgs}$. importés contre $9.046 \mathrm{kgs}$ exportés.

Anik-Devriès-Lesure é secretária geral da Revue de Musicologie, publicação da Société Française de Musicologie (Paris). 